OPEN ACCESS

Edited by: Guilhian Leipnitz,

Federal University of Rio Grande do

Sul, Brazil

Reviewed by:

Johan Palmfeldt,

Aarhus University, Denmark

Lina Ghaloul Gonzalez,

University of Pittsburgh, United States

*Correspondence:

Guenter Schwarz

gschwarz@uni-koeln.de

Specialty section:

This article was submitted to Genetics of Common and Rare

Diseases,

a section of the journal

Frontiers in Genetics

Received: 14 August 2020 Accepted: 30 November 2020 Published: 07 January 2021

Citation:

Mellis A-T, Roeper J, Misko AL, Kohl J and Schwarz G (2021) Sulfite Alters the Mitochondrial Network in Molybdenum Cofactor Deficiency.

Front. Genet. 11:594828

doi: $10.3389 /$ fgene.2020.594828

\section{Sulfite Alters the Mitochondrial Network in Molybdenum Cofactor Deficiency}

\author{
Anna-Theresa Mellis ${ }^{1}$, Juliane Roeper ${ }^{1}$, Albert L. Misko ${ }^{2}$, Joshua Kohl ${ }^{1}$ and \\ Guenter Schwarz",3*
}

\begin{abstract}
'Department of Chemistry, Institute for Biochemistry, University of Cologne, Cologne, Germany, ${ }^{2}$ Department of Neurology, Massachusetts General Hospital, Harvard Medical School, Boston, MA, United States, ${ }^{3}$ Center for Molecular Medicine, University of Cologne, Cologne, Germany
\end{abstract}

Molybdenum cofactor deficiency (MoCD) is an autosomal recessive disorder belonging to the large family of inborn errors in metabolism. Patients typically present with encephalopathy and seizures early after birth and develop severe neurodegeneration within the first few weeks of life. The main pathomechanism underlying MoCD is the loss of function of sulfite oxidase (SO), a molybdenum cofactor (Moco) dependent enzyme located in mitochondrial intermembrane space. SO catalyzes the oxidation of sulfite $\left(\mathrm{SO}_{3}{ }^{2-}\right)$ to sulfate $\left(\mathrm{SO}_{4}{ }^{2-}\right)$ in the terminal reaction of cysteine catabolism, and in the absence of its activity, sulfurous compounds such as $\mathrm{SO}_{3}{ }^{2-}$, S-sulfocysteine, and thiosulfate accumulate in patients. Despite growing evidence that these compounds affect neuronal and mitochondrial function, the molecular basis of neuronal dysfunction and cell death in MoCD is still poorly understood. Here we show that mitochondria are severely affected by the loss of SO activity. SO-deficient mouse embryonic fibroblasts display reduced growth rates and impaired ATP production when cultured in galactose, which is an indicator of mitochondrial dysfunction. We also found that mitochondria in SO-deficient cells form a highly interconnected network compared to controls while displaying a slight decrease in motility and unchanged mitochondrial mass. Moreover, we show that the mitochondrial network is directly influenced by $\mathrm{SO}_{3}{ }^{2-}$, as a moderate elevation of $\mathrm{SO}_{3}{ }^{2-}$ lead to the formation of an interconnected mitochondrial network, while high $\mathrm{SO}_{3}{ }^{2-}$ levels induced fragmentation. Finally, we found a highly interconnected mitochondrial network in MoCD patient-derived fibroblasts, similar to our findings in mouse-derived fibroblasts. We therefore conclude that altered mitochondrial dynamics are an important contributor to the disease phenotype and suggest that MoCD should be included among the mitochondrial disorders.

Keywords: sulfite, sulfite oxidase, mitochondria, molybdenum cofactor deficiency, fission and fusion

\section{INTRODUCTION}

Mitochondrial sulfite oxidase (SO) catalyzes the terminal step in the catabolism of cysteine and methionine, the oxidation of toxic sulfite $\left(\mathrm{SO}_{3}{ }^{2-}\right)$ to sulfate $\left(\mathrm{SO}_{4}{ }^{2-}\right)$, thereby passing two electrons to cytochrome $c$ (Johnson and Rajagopalan, 1979). The cellular concentration of cysteine is tightly controlled by its synthesis and degradation (Stipanuk and Ueki, 2011), the latter proceeding 
through two distinct pathways. The first, often referred to as the oxidative pathway, yields taurine, and $\mathrm{SO}_{4}{ }^{2-}$ as the main end products (Kohl et al., 2018). The second pathway involves enzymatic reactions that regulate the formation and clearance of $\mathrm{H}_{2} \mathrm{~S}$, an important signaling molecule in mammals, and terminates in the production of thiosulfate and $\mathrm{SO}_{4}{ }^{2-} \cdot \mathrm{SO}_{3}{ }^{2-}$ is a common metabolic intermediate in both catabolic pathways, that, if not removed by SO, can lead to various toxic effects (Vincent et al., 2004; Zhang et al., 2004; de Moura Alvorcem et al., 2017).

In man, the inherited loss of SO activity is caused by two different genetic mechanisms. First, SO may be impaired by mutations in the SUOX gene, thus leading to isolated sulfite oxidase deficiency (ISOD). Second, SO may be compromised by mutations in the molybdenum cofactor (Moco) biosynthetic genes (MOCS1, MOCS2, MOCS3, GPHN), thereby leading to Moco deficiency (MoCD) and loss of the Moco dependent SO activity (Kohl et al., 2018). Typically, MoCD and ISOD patients present in the neonatal period with identical clinical phenotypes, including encephalopathy, intractable seizures, feeding difficulties, and movement abnormalities. Disease progression involves psychomotor retardation due to progressive cerebral atrophy and ventricular dilatation, often resulting in fatal outcome within their first years of life (Schwarz, 2016). The most prominent biochemical hallmark of MoCD and ISOD is the accumulation of $\mathrm{SO}_{3}{ }^{2-}$ and the sulfite-cysteine adduct S-sulfocysteine in patient urine or plasma (Mudd et al., 1967). However, the disorders are distinguishable on the biochemical level due to the accumulation of xanthine and hypoxanthine, substrates of xanthine oxidase, another Moco enzyme, and diminished levels of uric acid in MoCD, but not ISOD (Schwarz et al., 2009; Schwahn et al., 2015).

Recently, we discovered a new mechanism underlying the neurodegeneration in SO deficiencies as we found that S-sulfocysteine can act as an NMDA receptor agonist and therefore leads to excitotoxic neuronal cell death (Kumar et al., 2017). $\mathrm{SO}_{3}{ }^{2-}$ toxicity has also been investigated in multiple studies, which indicate a disturbance of mitochondrial functions in neurons and kidney cells (Vincent et al., 2004; Zhang et al., 2004; Grings et al., 2014). In particular, exogenous $\mathrm{SO}_{3}{ }^{2-}$ decreased intracellular ATP levels, impaired cellular respiration and inhibited the mitochondrial enzymes glutamate dehydrogenase and malate dehydrogenase. Recently, impairment of mitochondrial respiration has also been shown in various patient cell lines with defects in cysteine catabolism (Grings et al., 2019).

Mitochondria are dynamic organelles constantly undergoing the processes of fusion and fission (Wai and Langer, 2016). The balance of fusion and fission can be influenced in either direction by a multitude of different factors including cellular respiration, ROS formation or the metabolic state of the cell, causing mitochondria to become hypertubular or fragmented. While the effect of $\mathrm{SO}_{3}{ }^{2-}$ on cellular bioenergetics and mitochondrial enzymes has been investigated before, the influence of $\mathrm{SO}_{3}{ }^{2-}$ on mitochondrial dynamics and morphology remains to be determined. In this study, we used $\mathrm{SO}$ deficient $\left(\right.$ Suox ${ }^{-/-}$) mouse embryonic fibroblast (MEF) cells to characterize mitochondrial morphology and function in SO deficiencies to shed light on the role of mitochondria in the disease pathology of ISOD and MoCD.

\section{MATERIALS AND METHODS}

\section{Cell Culture}

Mouse embryonic fibroblast cells were harvested from heterozygous breedings of Suox ${ }^{ \pm}$mice (Kohl et al., unpublished) as described previously ( $\mathrm{Xu}, 2005)$. In brief, embryos were removed at E13.5 after timed matings and dissected from the uterus. Fetuses were then minced into fine pieces and incubated in $0.25 \%$ trypsin-EDTA at $4^{\circ} \mathrm{C}$ overnight. On the next day, the suspension was heated for $30 \mathrm{~min}$ in a $37^{\circ} \mathrm{C}$ water bath. The digested tissue was then further broken down into a cell suspension by vigorous pipetting in culture medium. MEF cells were cultivated in Dulbecco's Modified Eagle Medium (DMEM) (Pan Biotech), supplemented with $2 \mathrm{mM}$ glutamine (Gibco by Life Technologies) and 10\% Fetal Bovine Serum (FBS) (Pan Biotech; Origin: South Africa). For galactose treatment, cells were cultured in glucose-free DMEM (Pan Biotech, P04-01548S1) supplemented with $10 \mathrm{mM}$ galactose or equal amounts of glucose as control.

Human fibroblasts were extracted from juvenile foreskin. Control fibroblasts were purchased from PromoCell (C-12300). Patient fibroblasts were kindly provided by J. Reiss (Institute for Human Genetics, University of Göttingen, Germany). GPHN deficient fibroblasts were first described in Reiss et al. (2001). MOCS1-deficient fibroblast were part of an earlier study (Reiss and Hahnewald, 2011). MOCS2 deficient fibroblasts were characterized in Hahnewald et al. (2006). Fibroblasts were kept in RPMI 1640 medium (Pan Biotech) supplemented with $2 \mathrm{mM}$ glutamine (Gibco by Life Technologies) and 10\% FBS (Pan Biotech; Origin: South Africa). All cells were cultured at $37^{\circ} \mathrm{C}$ and $5 \% \mathrm{CO}_{2}$.

\section{Mouse Keeping}

All animals were kept and bred in accordance with European, national and institutional guidelines and protocols were approved by local government authorities (Landesamt für Natur, Umwelt und Verbraucherschutz Nordrhein-Westfalen, Germany; reference 84-02.04.2014.A372). Mice were kept under a $12 \mathrm{~h}$ light cycle and provided with regular chow diet and water ad libitum. For the generation of homozygous Suox ${ }^{-/-}$mice, heterozygous mice of at least 2 months of age were kept in 1:1 (male:female) breedings.

\section{Mitochondrial Morphology Analysis}

Mitochondrial morphology in MEF cells and patient fibroblasts was visualized using MitoTracker ${ }^{\mathrm{TM}}$ Red CMXRos (Invitrogen, United States). The cells were seeded onto sterile cover slips the day prior to the experiment. Cells were incubated in 200 nM MitoTracker ${ }^{\mathrm{TM}}$ Red CMXRos diluted in fresh DMEM for $30 \mathrm{~min}$ at $37^{\circ} \mathrm{C}$. For $\mathrm{SO}_{3}{ }^{2-}$ treatments, the respective $\mathrm{SO}_{3}{ }^{2-}$ concentration was added to the medium $30 \mathrm{~min}$ prior to the MitoTracker and incubated at $37^{\circ} \mathrm{C}$. Afterward, the cells were washed carefully with PBS before fixation in 4\% PFA for $15 \mathrm{~min}$ 
at $4^{\circ} \mathrm{C}$. Remaining PFA was removed by three washing steps with PBS $(3 \times 5 \mathrm{~min})$. Finally, cover slips were mounted on slides with Mowiol/DABCO (Carl Roth). Images were acquired using a Nikon A1 confocal laser scanning microscope. Images were processed using ImageJ software.

\section{Mitochondrial Membrane Potential}

Mitochondrial membrane potential was assessed as described previously with minor modifications (Verburg and Hollenbeck, 2008). In short, a $5 \mu \mathrm{M}$ stock of tetramethylrhodamine methyl ester (TMRM, Invitrogen) in DMSO was diluted into DMEM medium to a final concentration of $20 \mathrm{nM}$. Cells were incubated in $20 \mathrm{nM}$ TMRM for $20 \mathrm{~min}$ at $37^{\circ} \mathrm{C}$. As a control, cells were co-stained with 200 nM MitoTracker ${ }^{\text {TM }}$ Deep Red (which accumulates in mitochondria independent of their membrane potential) for $20 \mathrm{~min}$ at $37^{\circ} \mathrm{C}$. Images were acquired with a Nikon A1R confocal microscope. Pixel intensity for the TMRM was subsequently quantified using ImageJ (Image), RRID:SCR_003070) and normalized by the respective intensity of the MitoTracker ${ }^{\mathrm{TM}}$ Deep Red staining.

\section{Mitochondrial Motility and Content}

The day prior to the experiment, cells were seeded onto a Nunc $^{\text {TM }}$ glass-bottom dish (Thermo Scientific, United States). Mitochondria were visualized via incubation with $200 \mathrm{nM}$ MitoTracker ${ }^{\text {TM }}$ Deep Red (diluted in DMEM) for $20 \mathrm{~min}$ at $37^{\circ} \mathrm{C}$. Mitochondrial motility was analyzed via life cell imaging using a Nikon A1R confocal microscope with environmental chamber. Time-lapse videos were taken over $5 \mathrm{~min}$ at $37^{\circ} \mathrm{C}$ and $5 \% \mathrm{CO}_{2}$. Mitochondrial motility and content were quantified after creating binary images with the software ImageJ (ImageJ, RRID:SCR_003070) as described in Koopman et al. (2006). In brief, maximum intensity projections of image stacks were converted to 8-bit formats and then subjected to a white tophat filter (MorphoLibJ plugin) before a final thresholding step. Finally, the amount of moved pixels of selected mitochondria between the first and last timepoint (always 5 min apart) were assessed using the XOR function of the Image Calculator.

\section{Transmission Electron Microscopy}

For Transmission electron microscopy (TEM), MEF cells were cultured on aclar foil and fixed with pre-warmed fixative solution (2\% glutaraldehyde, $2.5 \%$ sucrose, $3 \mathrm{mM} \mathrm{CaCl}_{2}, 100 \mathrm{Mm}$ HEPES, $\mathrm{pH} 7.3$ ) at RT for $30 \mathrm{~min}$ and $4^{\circ} \mathrm{C}$ for another $30 \mathrm{~min}$. Afterward, fixed cells were washed with $0.1 \mathrm{M}$ sodium cacodylate buffer, incubated with $1 \% \mathrm{OsO}_{4}, 1.25 \%$ Sucrose, $10 \mathrm{mg} / \mathrm{ml}$ Potassium ferrocyanide in $0.1 \mathrm{M}$ Cacodylate buffer for $1 \mathrm{~h}$ on ice, and washed three times with $0.1 \mathrm{M}$ Cacodylate buffer. Subsequently, cells were dehydrated using ascending ethanol series $(50,70,90,3 \times 100)$ for $7 \mathrm{~min}$ each at $4^{\circ} \mathrm{C}$. After that, cells were incubated with ascending EPON series at $4^{\circ} \mathrm{C}$ : EPON with ethanol $(1+1)$ for $1 \mathrm{~h}$, EPON with ethanol $(3+1)$ for $2 \mathrm{~h}$, EPON alone ON, $2 \times 2 \mathrm{~h}$ with fresh EPON at RT and finally embedded for $48-72 \mathrm{~h}$ at $62^{\circ} \mathrm{C}$. Ultrathin sections of $70 \mathrm{~nm}$ were cut with a diamond knife using an ultramicrotome (Leica, UC7) and stained with uranyl acetate for $15 \mathrm{~min}$ at $37^{\circ} \mathrm{C}$ and lead nitrate solution for $4 \mathrm{~min}$. Electron micrographs were taken with a JEM-2100 Plus Electron
Microscope (JEOL). Camera OneView 4K 16 bit (Gatan), and software DigitalMicrograph (Gatan).

\section{ATP Measurement}

Intracellular ATP levels in MEF cells were quantified using the CellTiter-Glo ${ }^{\circledR}$ Luminescent Cell Viability Assay (Promega, United States). In short, $8 \times 10^{4}$ cells per well were seeded in 6-well plates the day prior to the experiment. The cells were incubated in glucose- or galactose containing medium for $1 \mathrm{~h}$. They were then lysed in $150 \mu \mathrm{l}$ RIPA buffer (Sigma). After centrifugation for $20 \mathrm{~min}$ at $4^{\circ} \mathrm{C}$ and $13,000 \mathrm{~g}, 50 \mu \mathrm{l}$ of the lysate was added to $50 \mu \mathrm{l}$ CellTiter-Glo ${ }^{\circledR}$ Reagent in a 96-well. The plate was placed on an orbital shaker for $2 \mathrm{~min}$ to $\mathrm{mix}$ the contents before a $10 \mathrm{~min}$ incubation at room temperature. Luminescence was recorded using an EnVision Multimode Plate Reader (PerkinElmer, United States). ATP levels were quantified by comparison to a standard curve and normalized to the protein concentration in the respective sample.

\section{Cell Viability Assay}

Cell viability after incubation in glucose- or galactose containing medium was assessed by the MTT assay as described previously (Kumar et al., 2017). In brief, $1 \times 10^{4}$ cells/well were seeded onto a 96-well and grown for $24 \mathrm{~h}$. The next day, the medium was exchanged for DMEM containing either $10 \mathrm{mM}$ glucose or galactose as a carbon source. After $24 \mathrm{~h}$, the old medium was exchanged with fresh phenol red- free DMEM containing $100 \mu \mathrm{M}$ (3-(4,5-dimethylthiazol-2-yl)-2,5-diphyenyltetrazolium bromide) (MTT). After a $4 \mathrm{~h}$ incubation at $37^{\circ} \mathrm{C}$ in the dark, the MTT solution was exchanged for $100 \%$ DMSO. The plate was then placed in a shaker for $10-30 \mathrm{~min}$ at $37^{\circ} \mathrm{C}$ until all cells were solubilized. Finally, the absorption was measured at $570 \mathrm{~nm}$ (reference $650 \mathrm{~nm}$ ) in well plate reader (Tecan Spark).

\section{Determination of Protein Concentration}

The concentration of proteins from cell extracts was determined by means of the Bradford assay. Therefore, $190 \mu$ l diluted Bradford solution (1/5 dilution) was incubated with $10 \mu \mathrm{l}$ of protein solution (respective dilution) for $20 \mathrm{~min}$ and the absorption change at $595 \mathrm{~nm}$ was measured using a well plate reader (BioTek, Germany). The determined absorption change was then compared to that of standard proteins with known concentration in order to determine the protein concentration of unknown solutions.

\section{Western Blot Analysis}

Western blotting was performed on crude protein extracts from MEF cells lysed in $100 \mathrm{mM}$ Tris/Ac, $\mathrm{pH}$ 8.0. Protein concentration was adjusted accordingly after determination with the Bradford assay. Unless otherwise indicated, $30 \mu \mathrm{g}$ of protein lysate were separated by SDS-PAGE and immunoblotted using a standard semi-dry blotting protocol onto PVDF membranes. SO protein levels were visualized using a monoclonal anti-SUOX antibody (Sigma-Aldrich Cat\# WH0006821M1, RRID:AB_1843810) in conjunction with a HRP-coupled anti-mouse secondary antibody (Santa Cruz 
Biotechnology, United States Cat\# sc-2055, RRID:AB_631738). Vinculin was detected as loading control using a polyclonal anti-vinculin (H-300) antibody (Santa Cruz Biotechnology, United States Cat\# sc-5573, RRID:AB_2214507) together with a HRP-coupled anti-rabbit secondary antibody (Santa Cruz Biotechnology, United States Cat\# sc-2054, RRID:AB_631748). Signals were detected using chemiluminescent substrates (Thermo Fisher Scientific, United States, \#34580) and a Bio-Rad ChemiDoc XRS + system.

\section{RESULTS}

\section{Suox ${ }^{-/-}$MEFs Show Mitochondrial Impairment}

Since previous studies indicate that $\mathrm{SO}_{3}{ }^{2-}$ impacts mitochondrial function (Vincent et al., 2004; Zhang et al., 2004; Grings et al., 2014), we first tested whether SO-deficient MEF cells (Figure 1A) generated from Suox ${ }^{-/-}$mice (Kohl et al. unpublished results) exhibited signs of mitochondrial damage. We cultured WT and Suox ${ }^{-/-}$MEFs in either glucoseor galactose-containing medium, thereby forcing the cells to rely on oxidative phosphorylation (OXPHOS) as the main energy source in absence of glucose (galactose-containing medium). After growing the cells for $24 \mathrm{~h}$, we analyzed cell survival using the MTT assay (Figure 1B). While the absence of glucose had no effect on the viability of WT cells, survival of Suox ${ }^{-/}$cells was reduced to less than $50 \%$ in galactose, which indicates mitochondrial damage. We then measured intracellular ATP levels under the same conditions and again found no effect on WT MEFs, while Suox ${ }^{-/-}$cells exhibited a severe reduction of ATP levels in galactose-containing medium ( $~ 20 \%$ of control), thus showing that Suox ${ }^{-/-}$MEFs are impaired in their ability to produce ATP via OXPHOS (Figure 1C). An essential component of OXPHOS is the generation of a proton gradient by different complexes of the respiratory chain (I, III, and IV). We therefore analyzed the mitochondrial membrane potential in WT and Suox ${ }^{-/-}$cells via TMRM staining and found that Suox ${ }^{-/-}$ cells have a mild, but significant reduction in their membrane potential relative to WT (Figure 1D).

\section{Mitochondrial Morphology Is Altered in SuOx ${ }^{-/-}$MEFs}

Next, we visualized mitochondrial morphology in WT and Suox ${ }^{-/}$cells after staining with MitoTracker Red CMXRos (Figure 2A). We observed that mitochondria in Suox ${ }^{-/-}$MEFs formed a tightly interconnected and hypertubular network distinct from WT MEF cells. We quantified 100 cells per genotype and categorized the mitochondrial network as either fragmented (disturbed, dot-like network, almost no tubules
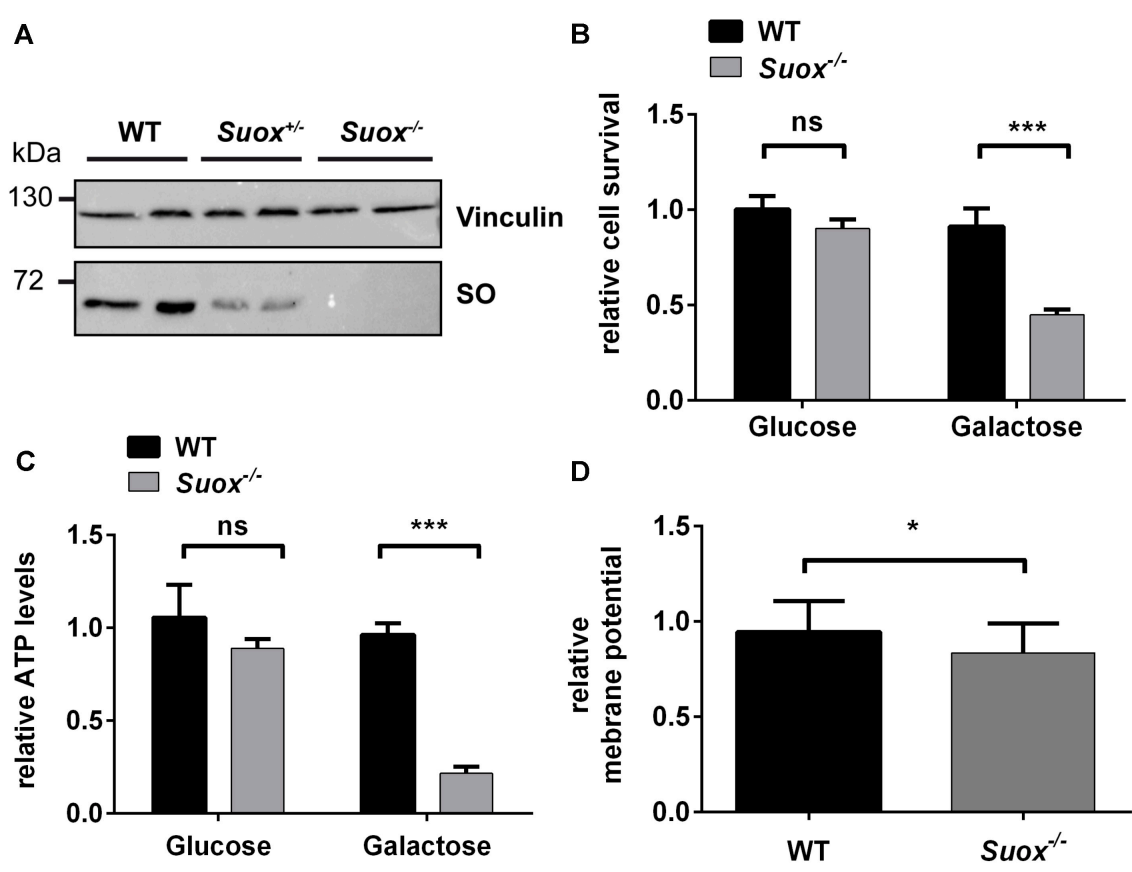

D

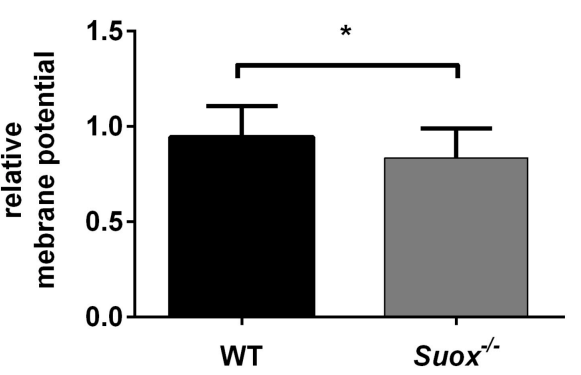

FIGURE 1 | Mitochondrial deficiency in Suox ${ }^{-/-}$MEFs. (A) Sulfite oxidase (SO) protein expression in WT, Suox ${ }^{ \pm}$and Suox ${ }^{-/-}$MEFs. Vinculin was used as a loading control. (B) Determination of cell survival of WT and Suox ${ }^{-/}$MEFs after a $24 \mathrm{~h}$ incubation in glucose- or galactose containing DMEM medium $(n=6)$. Cell viability was assessed using the MTT assay (Promega). Values were normalized to WT grown in glucose medium. (C) Measurement of cellular ATP levels of WT and Suox ${ }^{-1-}$ MEFs after a $24 \mathrm{~h}$ incubation in glucose- or galactose containing DMEM $(n=3)$. Intracellular ATP levels were quantified using the CellTiter-Glo Luminescent Cell Viability Assay (Promega). Values were normalized to WT grown in glucose medium. (D) Determination of mitochondrial membrane potential in WT and Suox ${ }^{-/-}$ MEFs $(n=3)$. Mitochondrial membrane potential was assessed by staining with $20 \mathrm{nM}$ TMRM (membrane potential-dependent). The pixel intensity was quantified relative to $200 \mathrm{nM}$ MitoTracker Deep Red staining (membrane potential independent). Values were normalized to WT measurement. (B-D): Error bars indicate standard deviation. Student's $t$ test was performed as indicated. $p$ value: ${ }^{\star \star \star}<0.001 ;{ }^{* \star}<0.01 ;{ }^{*}<0.05 ; \mathrm{ns}>0.05$. 
A
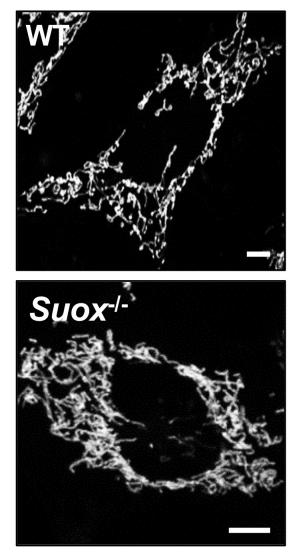

C
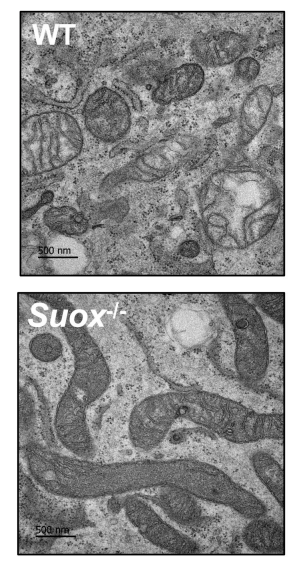
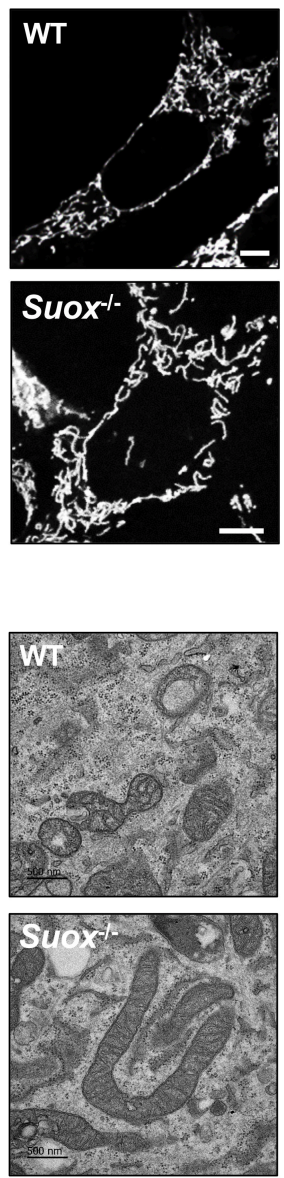

B

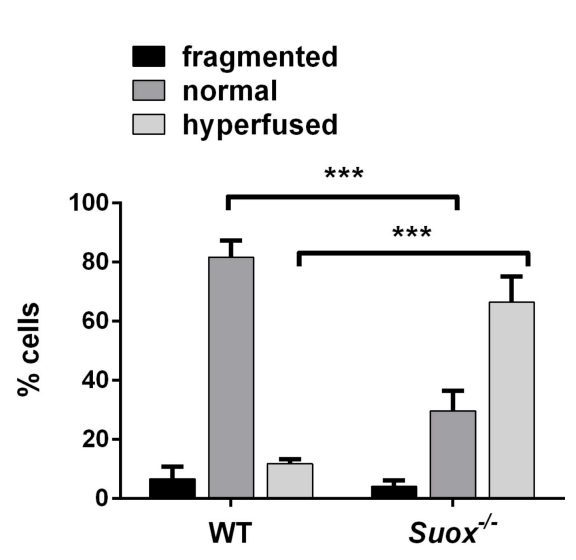

D

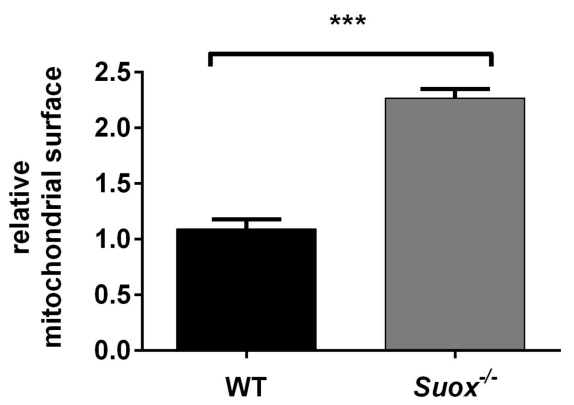

FIGURE 2 | Mitochondrial morphology in Suox ${ }^{-/-}$MEFs. (A) Visualization of the mitochondrial network in WT and Suox ${ }^{-/-}$MEFs. Mitochondrial networks in WT and Suox ${ }^{-/-}$MEFs were visualized by incubation in $200 \mathrm{nM}$ MitoTracker Red CMXRos (Invitrogen) for $30 \mathrm{~min}$ at $37^{\circ} \mathrm{C}$. Suox ${ }^{-/-}$MEFs show a more interconnected and elongated mitochondrial network than WT cells. Scale-bar represents $10 \mu \mathrm{m}$. (B) Quantification of mitochondrial network phenotypes in WT and Suox ${ }^{-/-}$MEFs presented in panel (A). 100 cells per genotype were categorized according to their mitochondrial network as either fragmented (disturbed, dot-like network, almost no tubules present), normal (both tubules and dot-like mitochondria present) or hyperfused (highly interconnected tubules, no dot-like mitochondria present) ( $n=3$ ). Error bars indicate standard deviation. Ordinary two-way ANOVA with Tukey's post hoc test for pairwise comparisons was performed as indicated. $p$ value: ${ }^{* \star \star}$ $<0.001 ;^{* \star}<0.01 ;^{*}<0.05$; ns $>0.05$. (C) Mitochondria captured from WT and Suox ${ }^{-1-}$ MEFs via TEM. Suox ${ }^{-1-}$ MEFs show highly elongated mitochondria compared to WT cells. Scale bar represents $500 \mathrm{~nm}$. (D) Quantification of the mitochondrial surface area in WT and Suox ${ }^{-/-}$MEFs based on TEM pictures presented in (C). The surfaces of 50 individual mitochondria were measured with Image J and normalized to WT values $(n=3)$. Error bars indicate standard deviation. Student's $t$ test was performed as indicated. $p$ value: ${ }^{\star \star \star}<0.001 ;{ }^{* \star}<0.01 ;{ }^{*}<0.05 ;$ ns $>0.05$.

present) normal (both tubules and dot-like mitochondria present) or hyperfused (highly interconnected tubules, no dotlike mitochondria present). We found that for WT cells $81 \%$ had a normal mitochondrial network, while $6 \%$ displayed fragmented mitochondria, and 13\% were hyperfused (Figure 2B). In contrast, only $30 \%$ of the Suox ${ }^{-/-}$cells had a normal mitochondrial network, while $4 \%$ had a fragmented network, and $66 \%$ had a hyperfused network. To complement these light microscopic studies, we performed TEM and again found the mitochondria in the KO cells to be abnormally elongated compared to WT (Figure 2C). Quantification of the cross-sectional area of 50 individual mitochondria confirmed that mitochondria from Suox ${ }^{-/-}$MEFs were on average more than twice as large than WT mitochondria (Figure 2D). Finally, TEM revealed that the elongated mitochondria also showed a much more electron dense matrix structure than WT mitochondria.

\section{Suox-/- Mitochondria Are Less Motile Than WT}

Mitochondria have a high degree of motility, which enables them to distribute throughout the cytoplasm and facilitates both fusion and fission processes. To measure mitochondrial mobility, we acquired time-lapse videos of mitochondrial movements in an environmental chamber at $37^{\circ} \mathrm{C}$ over a period of $5 \mathrm{~min}$. The first and last image of each video were colored red or green and overlaid. Yellow pixels represented mitochondria that remained stationary over the 5 min period, while red and green 
pixels were quantified as a proxy measure of mitochondrial motility (Yi et al., 2004; Figure 3A). Our quantification showed that Suox ${ }^{-/-}$mitochondria moved less than WT mitochondria (Figure 3B). We also measured the total amount of mitochondria per cell and found no statistically significant difference, although Suox ${ }^{-/-}$cells tend to have less mitochondrial content than WT cells (Figure 3C).

\section{Sulfite Treatment Leads to Abnormal Mitochondrial Morphology}

We reasoned that the alterations in mitochondrial morphology observed in Suox ${ }^{-/-}$cells could be a direct result of intracellular $\mathrm{SO}_{3}{ }^{2-}$ accumulation. To test whether $\mathrm{SO}_{3}{ }^{2-}$ affects mitochondrial morphology, we treated human WT fibroblasts with escalating doses of $\mathrm{SO}_{3}{ }^{2-}$ concentrations and assessed for changes in mitochondrial morphology after $1 \mathrm{~h}$ of treatment (Figure 4A). Addition of 10 or $50 \mu \mathrm{M} \mathrm{SO}{ }^{2-}$ led to an increase in the percentage of cells with hyperfused mitochondria (Figure 4B). While we found that $\sim 11 \%$ of untreated WT cells were hyperfused, addition of 10 or $50 \mu \mathrm{M} \mathrm{SO}{ }^{2-}$ resulted in $\sim 32 \%$ and $23 \%$ hyperfused cells, respectively. We also observed that treatment with higher $\mathrm{SO}_{3}{ }^{2-}$ concentrations lead to a higher percentage of fragmented mitochondria. Around 50\% of the cells displayed fragmented mitochondria after treatment with $500 \mu \mathrm{M} \mathrm{SO}_{3}{ }^{2-}$, whereas untreated cells showed fragmented mitochondria in $\sim 4 \%$ of all analyzed cells. In turn, the number of cells categorized to harbor a normal mitochondrial network were significantly reduced in all $\mathrm{SO}_{3}{ }^{2-}$-treated cells, regardless of the respective concentration. While $84 \%$ of untreated cells had a normal mitochondrial network, this percentage was reduced to $\sim 57 \%$ for $\mathrm{SO}_{3}{ }^{2-}$ concentrations up to $100 \mu \mathrm{M}$ and $\sim 50 \%$ for 250 and $500 \mu \mathrm{M} \mathrm{SO}_{3}{ }^{2-}$ treatments. These data show that $\mathrm{SO}_{3}{ }^{2-}$ directly influences mitochondrial morphology in a dosedependent manner.

\section{MoCD Patient Derived Fibroblasts Recapitulate the Mitochondrial Phenotype of Suox ${ }^{-/-}$MEF Cells}

Finally, we analyzed mitochondrial morphology in fibroblasts derived from various MoCD patients that are impaired in

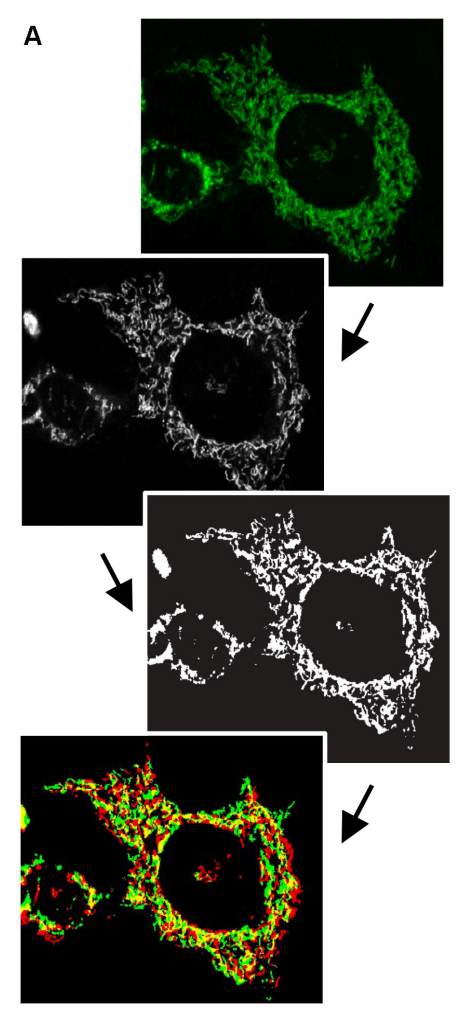

B

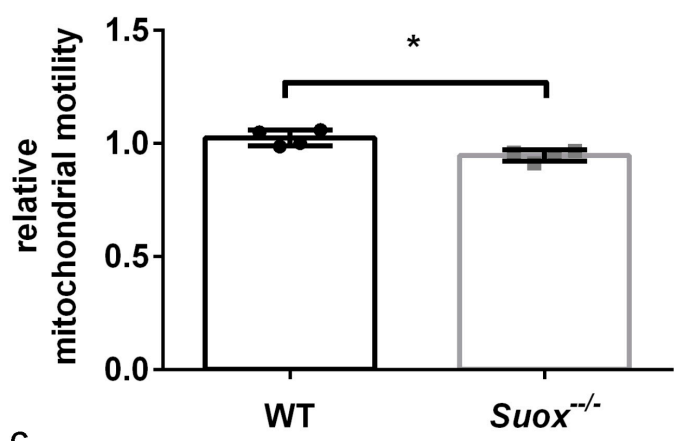

C

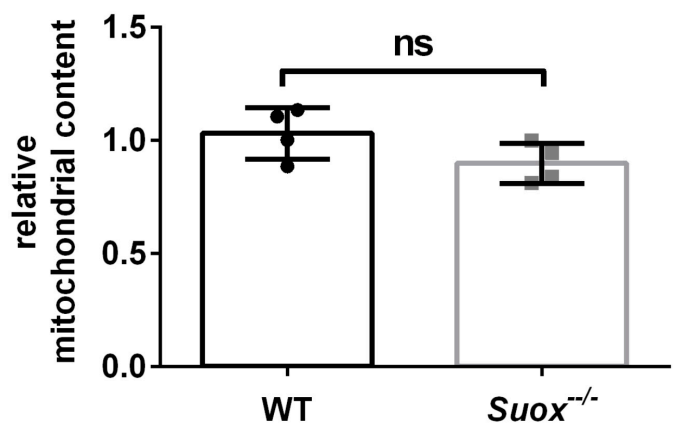

FIGURE 3 | Mitochondrial motility and content in Suox-/- MEFs. (A) Workflow of mitochondrial motility analysis using ImageJ. Starting from the left, maximum intensity projections of image stacks (first picture) were converted to 8-bit formats (second picture) and then subjected to a white top-hat filter (MorphoLibJ plugin), before a final thresholding step (third picture). Finally, the amount of moved pixels of selected mitochondria (last picture) between the first (green) and last (red) timepoint (always 5 min apart) were assessed using the XOR function of the Image Calculator with yellow pixel indicate overlapping pixels. (B) Quantification of mitochondrial motility as shown in (A). Mitochondrial motility was analyzed via life cell imaging following incubation with $200 \mathrm{nM}$ MitoTracker Deep Red (diluted in DMEM) for $20 \mathrm{~min}$ at $37^{\circ} \mathrm{C}$. Values were normalized to WT. Error bars indicate standard deviation. Student's $t$ test was performed as indicated. $p$ value: ${ }^{\star \star \star}<0.001$; ${ }^{* *}<0.01$; ${ }^{*}<0.05$; ns $>0.05$. (C) Quantification of mitochondrial content. Mitochondria were visualized via incubation with $200 \mathrm{nM}$ MitoTracker Deep Red (diluted in DMEM) for 20 min at $37^{\circ} \mathrm{C}$. Total amount of pixels per cell was quantified using ImageJ. Values were normalized to WT. Error bars indicate standard deviation. Student's $t$ test was performed as indicated. $p$ value: ${ }^{* \star *}<0.001 ;{ }^{* \star}<0.01 ;{ }^{*}<0.05 ; \mathrm{ns}>0.05$. 


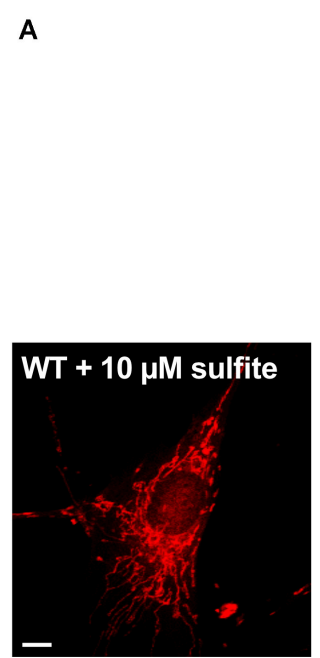

C
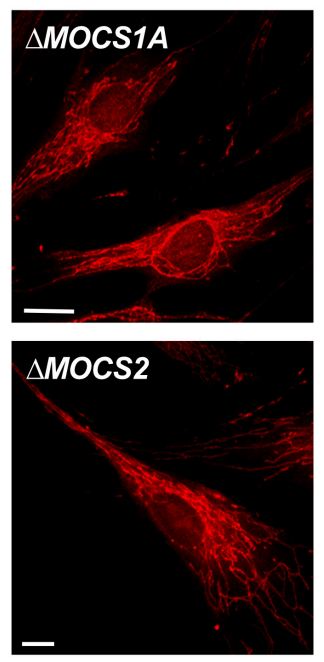
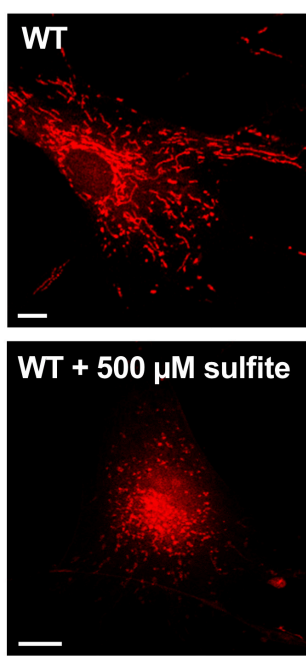

\section{B $\square$ fragmented \\ $\square$ normal \\ hyperfused}

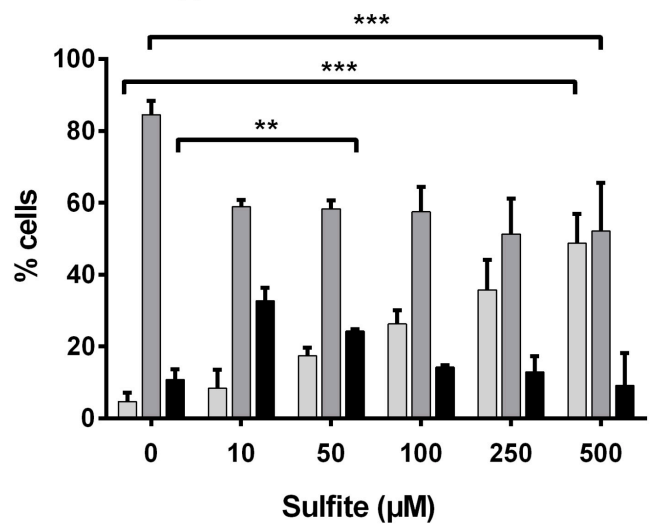

D
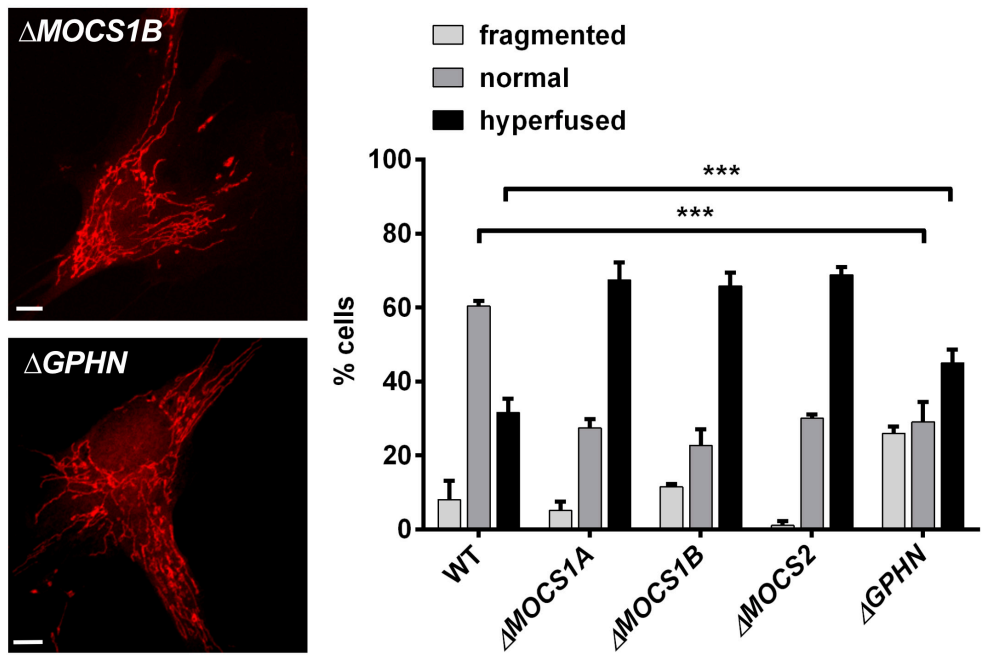

FIGURE 4 | Influence of sulfite $\left(\mathrm{SO}_{3}{ }^{2-}\right)$ on mitochondrial morphology. (A) Imaging of the mitochondrial network in human WT fibroblasts with and without $\mathrm{SO}_{3}{ }^{2-}$. Mitochondria in fibroblasts were visualized using $200 \mathrm{nM}$ MitoTracker Red CMXRos (Invitrogen) for $30 \mathrm{~min}$ at $37^{\circ} \mathrm{C} \mathrm{For} \mathrm{SO}_{3}{ }^{2-}$ treatments, the respective $\mathrm{SO}_{3}{ }^{2-}$ concentration was added to the medium 30 min prior to the MitoTracker and incubated at $37^{\circ} \mathrm{C}$. While untreated WT cells show a normal mitochondrial network

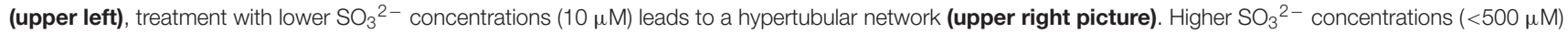
result in mitochondrial fragmentation (lower left picture). Scale-bar represents $10 \mu \mathrm{m}$. (B) Quantification of mitochondrial network phenotypes in WT fibroblasts after treatment with different $\mathrm{SO}_{3}{ }^{2-}$ concentrations as presented in (A). 100 cells per genotype were categorized according to their mitochondrial network as either fragmented (disturbed, dot-like network, almost no tubules present), normal (both tubules and dot-like mitochondria present) or hyperfused (highly interconnected tubules, no dot-like mitochondria present, length) $(n=3)$. Error bars indicate standard deviation. Ordinary two-way ANOVA with Tukey's post hoc test for pairwise comparisons was performed as indicated. $p$ value: ${ }^{* \star}<0.001$; ${ }^{* *}<0.01$; ${ }^{*}<0.05$; ns $>0.05$. (C) Imaging of the mitochondrial network in fibroblasts from different MoCD patients. Mitochondria in different patient fibroblasts were visualized using $200 \mathrm{nM}$ MitoTracker Red CMXRos (Invitrogen) for 30 min at $37^{\circ} \mathrm{C}$. All cell lines presented strongly interconnected and hyperfused mitochondria. Scale-bar represents $10 \mu \mathrm{m}$. (D) Quantification of mitochondrial network phenotypes in fibroblasts from different MoCD patients presented in (C). 100 cells per genotype were categorized according to their mitochondrial network as either fragmented (disturbed, dot-like network, almost no tubules present), normal (both tubules and dot-like mitochondria present) or hyperfused (highly interconnected tubules, no dot-like mitochondria present) $(n=3)$. Error bars indicate standard deviation. Ordinary two-way ANOVA with Tukey's post hoc test for pairwise comparisons was performed as indicated. $p$ value: ${ }^{* \star *}<0.001 ;{ }^{* *}<0.01 ;{ }^{*}<0.05 ; \mathrm{ns}>0.05$.

different genes important for Moco biosynthesis, namely MOCS1A, MOCS1B, MOCS2, and GPHN (Figure 4C). We found that cell lines derived from patients with disease causing mutations in MOCS1 or MOCS2 70\% contained a hyperfused mitochondrial network, whereas this was only the case for $30 \%$ of the cells for WT fibroblasts (Figure 4D). In cells derived from patients with disease causing mutations in the GPHN gene, $45 \%$ were characterized as hyperfused. In turn, $\sim 60 \%$ of WT cells displayed a normal mitochondrial network, while this phenotype could only be found in $\sim 25-30 \%$ of all patient cells 
regardless of the affected gene. The percentage of cells with a fragmented mitochondrial network varied between $1 \%$ and $10 \%$ for the MOCS1/2 and WT cell lines. Remarkably, only $1 \%$ of the cells deficient in MOCS2 were fragmented, whereas $25 \%$ of the cells affected in GPHN showed fragmented mitochondria. In summary, these data confirm a disturbed mitochondrial network in different patient cell lines of MoCD that can collectively be traced back to $\mathrm{SO}_{3}{ }^{2-}$ accumulation.

\section{DISCUSSION}

Sulfite oxidase is a mitochondrial enzyme that is vital for the detoxification of $\mathrm{SO}_{3}{ }^{2-}$. Inactivation of $\mathrm{SO}$, in either ISOD or MoCD, leads to severe neurodegeneration, often with lethal outcome. While mitochondrial impairment has been observed following $\mathrm{SO}_{3}{ }^{2-}$ treatment of different model systems (Vincent et al., 2004; Zhang et al., 2004; Grings et al., 2013), the underlying mechanism and role of mitochondrial damage within the context of SO deficiency remains poorly understood. In this study, we show that mitochondria in Suox ${ }^{-/-}$and patient fibroblasts are abnormally elongated and interconnected. Application of exogenous $\mathrm{SO}_{3}{ }^{2-}$ on WT fibroblasts induced similar morphological changes in the mitochondrial network, suggesting that $\mathrm{SO}_{3}{ }^{2-}$ accumulation in SO-deficient cells directly mediates alterations in mitochondrial function. This pathomechanism provides a new concept underlying $\mathrm{SO}_{3}{ }^{2-}$ toxicity in the SO deficiencies.

We found that cellular viability and ATP production under galactose treatment was markedly reduced in Suox ${ }^{-/-}$MEFs, indicating that the mitochondria in those cells are dysfunctional and thus unable to produce sufficient ATP via OXPHOS. In line with this, one of the earliest identified toxic effects of $\mathrm{SO}_{3}{ }^{2-}$ was a dose-dependent reduction of total cellular ATP levels (Zhang et al., 2004). Moreover, direct treatment of $\mathrm{SO}_{3}{ }^{2-}$ also decreased ATP production from mitochondria isolated from rat renal epithelial cells (Vincent et al., 2004). Therefore, it is likely that elevated $\mathrm{SO}_{3}{ }^{2-}$ levels, due to the lack of $\mathrm{SO}$, lead to the inhibition of mitochondrial ATP production and reduced viability upon longer cultivation in galactose.

In a recent study, Grings et al. (2019) reported that MOCS1 patient fibroblasts display altered protein levels of mitofusins 1 and 2, indicating a disturbance of mitochondrial dynamics. We analyzed the mitochondrial network in different cell culture models of SO deficiency and found that the mitochondria were hyperfused in the vast majority of SO-deficient cells. Furthermore, treatment of WT cells with low $\mathrm{SO}_{3}{ }^{2-}$ concentrations (up to $40 \mu \mathrm{M}$ ) resulted in similar morphological changes, suggesting that the observed abnormalities are directly mediated by $\mathrm{SO}_{3}{ }^{2-}$. Hyperfusion is known as a typical response to mild mitochondrial stress, for example nutrient starvation, as it allows to separate mitochondria within one cell to exchange their contents and thereby help to "recover" individual damaged mitochondria (Rugarli et al., 2012). However, treatment of WT cells with high $\mathrm{SO}_{3}{ }^{2-}$ concentrations (more than $50 \mu \mathrm{M}$ ) lead to increased mitochondrial fragmentation, indicating that at this point, the mitochondrial damage becomes too severe to be repaired. Mitochondrial fragmentation can be induced by various different forms of stress and allows the removal of terminally damaged mitochondria by mitophagy (Rugarli et al., 2012). In particular, inhibition of the mitochondrial enzymes malate dehydrogenase and glutamate dehydrogenase has been shown for $\mathrm{SO}_{3}{ }^{2-}$ concentrations of $100 \mu \mathrm{M}$ and higher (Zhang et al., 2004). Inhibition of the central metabolic enzyme glutamate dehydrogenase could lead to a decreased metabolic flux through the TCA cycle, which might further contribute to the reduced ATP production, thereby inducing fragmentation.

Hyperfusion of mitochondria may be induced by an increase in fusion or a decrease in fission processes. We detected a decrease in mitochondrial motility in Suox ${ }^{-/-}$cells, which might suggest a decrease in fission. Correct function of Drp1, a GTPase that mediates mitochondrial fission, is regulated by a number of different mechanisms, such as recruitment to the outer mitochondrial membrane by recruiting factors as well as post-translational modifications such as phosphorylation or nitrosylation (Suárez-Rivero et al., 2016). Following our initial findings, further studies are needed to elucidate the exact mechanism of how $\mathrm{SO}_{3}{ }^{2-}$ alters mitochondrial dynamics.

The most affected organ in the SO deficiencies is the nervous system. Interestingly, this is also the case in many other disorders of disrupted mitochondrial dynamics. Both Drp1 and mitofusin2 are required for neuronal development and dysregulation of either protein is associated with a neurological disorder (SuárezRivero et al., 2016). For example, mitofusin-2 is necessary for cerebellar development in mice (Chen et al., 2007), and dominant negative variants of mitofusin-2 are associated with Charcot-Marie-Tooth disease type 2A, a length dependent peripheral neuropathy in man (Kijima et al., 2005). Drp1 on the other hand has been associated with multiple disorders, including Huntington's disease, amyotrophic lateral sclerosis, and Parkinson's disease (Song et al., 2011, 2013; Wang et al., 2011).

Among the group of mitochondrial disorders, there is another disease of sulfur metabolism, called ethylmalonic encephalopathy or ETHE1 deficiency (Tiranti et al., 2004). Similar to SO deficiencies, patients present with early onset progressive neurological degeneration, psychomotor retardation and excretion of ethylmalonic acid in urine, resulting in death in the first few years of life (Tiranti et al., 2004). ETHE1 patients accumulate high levels of $\mathrm{H}_{2} \mathrm{~S}$ and thiosulfate in biological fluids and tissues, the latter also being elevated in ISOD and MoCD. Intriguingly, $\mathrm{H}_{2} \mathrm{~S}$ has been reported to interfere with cellular respiration via inhibition of cytochrome $\mathrm{c}$ oxidase (Tiranti et al., 2009). First attempts have recently been made to improve mitochondrial respiratory function in fibroblasts from ETHE1 and MOCS1 patients by treating cells with the mitochondria-targeted antioxidant JP4-039 (Grings et al., 2019). While these results are promising, future studies should aim for further dissection of the underlying molecular mechanisms that primarily drive mitochondrial pathology in neuronal tissues of SO deficiency models in order to provide new therapy options. In summary, we show here that mitochondrial dynamics are impaired by $\mathrm{SO}_{3}{ }^{2-}$ and dysregulated in $\mathrm{SO}$ deficiencies, thereby providing further evidence that SO deficiencies join the large and diverse family of mitochondrial disorders. 


\section{DATA AVAILABILITY STATEMENT}

The raw data supporting the conclusions of this article will be made available by the authors, without undue reservation.

\section{ETHICS STATEMENT}

All animals were kept and bred in accordance with European, national and institutional guidelines and protocols were approved by local government authorities (Landesamt für Natur, Umwelt und Verbraucherschutz Nordrhein-Westfalen, Germany; reference 84-02.04.2014.A372).

\section{AUTHOR CONTRIBUTIONS}

A-TM, JR, and GS: study design. JK: providing of study materials. A-TM, JR, AM, and GS: data generation, analysis, and interpretation. A-TM, AM, and GS: manuscript writing.

\section{REFERENCES}

Chen, H., McCaffery, J. M., and Chan, D. C. (2007). Mitochondrial fusion protects against neurodegeneration in the cerebellum. Cell 130, 548-562. doi: 10.1016/J. CELL.2007.06.026

de Moura Alvorcem, L., da Rosa, M. S., Glänzel, N. M., Parmeggiani, B., Grings, M., Schmitz, F., et al. (2017). Disruption of energy transfer and redox status by sulfite in hippocampus, striatum, and cerebellum of developing rats. Neurotox. Res. 32, 264-275. doi: 10.1007/s12640-017-9732-y

Grings, M., Moura, A. P., Amaral, A. U., Parmeggiani, B., Gasparotto, J., Moreira, J. C. F., et al. (2014). Sulfite disrupts brain mitochondrial energy homeostasis and induces mitochondrial permeability transition pore opening via thiol group modification. Biochim. Biophys. Acta 1842, 1413-1422. doi: 10.1016/j.bbadis. 2014.04.022

Grings, M., Moura, A. P., Parmeggiani, B., Marcowich, G. F., Amaral, A. U., de Souza Wyse, A. T., et al. (2013). Disturbance of brain energy and redox homeostasis provoked by sulfite and thiosulfate: potential pathomechanisms involved in the neuropathology of sulfite oxidase deficiency. Gene 531, 191-198. doi: 10.1016/j.gene.2013.09.018

Grings, M., Seminotti, B., Karunanidhi, A., Ghaloul-Gonzalez, L., Mohsen, A.W., Wipf, P., et al. (2019). ETHE1 and MOCS1 deficiencies: disruption of mitochondrial bioenergetics, dynamics, redox homeostasis and endoplasmic reticulum-mitochondria crosstalk in patient fibroblasts. Sci. Rep. 9:12651. doi: 10.1038/s41598-019-49014-2

Hahnewald, R., Leimkühler, S., Vilaseca, A., Acquaviva-Bourdain, C., Lenz, U., and Reiss, J. (2006). A novel MOCS2 mutation reveals coordinated expression of the small and large subunit of molybdopterin synthase. Mol. Genet. Metab. 89, 210-213. doi: 10.1016/j.ymgme.2006.04.008

Johnson, J. L., and Rajagopalan, K. V. (1979). The oxidation of sulphite in animals systems. Ciba Found. Symp. 1979, 119-133. doi: 10.1002/9780470720554.CH8

Kijima, K., Numakura, C., Izumino, H., Umetsu, K., Nezu, A., Shiiki, T., et al. (2005). Mitochondrial GTPase mitofusin 2 mutation in charcot-marie-tooth neuropathy type 2A. Hum. Genet. 116, 23-27. doi: 10.1007/s00439-004-1199-2

Kohl, J. B., Mellis, A.-T., and Schwarz, G. (2018). Homeostatic impact of sulfite and hydrogen sulfide on cysteine catabolism. Br. J. Pharmacol. 176, 554-570. doi: 10.1111/bph.14464

Koopman, W. J. H., Visch, H.-J., Smeitink, J. A. M., and Willems, P. H. G. M. (2006). Simultaneous quantitative measurement and automated analysis of mitochondrial morphology, mass, potential, and motility in living human skin fibroblasts. Cytom. Part A 69A, 1-12. doi: 10.1002/cyto.a.20198

Kumar, A., Dejanovic, B., Hetsch, F., Semtner, M., Fusca, D., Arjune, S., et al. (2017). S-sulfocysteine/NMDA receptor-dependent signaling underlies
All authors contributed to the article and approved the submitted version.

\section{FUNDING}

This work was supported by the German Research Foundation (Deutsche Forschungsgemeinschaft, DFG) SFB1218 project number 269925409 and SFB1403 project number 414786233.

\section{ACKNOWLEDGMENTS}

We acknowledge Simona Jansen, Monika Laurien, and Joana Stegemann (University of Cologne) for technical assistance. We thank Dr. Jochen Reiss (University of Göttingen) for providing the patient fibroblasts. We also thank the CECAD Imaging Facility, University of Cologne (Head: Dr. Astrid Schauss) for their support.

neurodegeneration in molybdenum cofactor deficiency. J. Clin. Invest. 127, 4365-4378. doi: 10.1172/JCI89885

Mudd, S. H., Irreverre, F., and Laster, L. (1967). Sulfite oxidase deficiency in man: demonstration of the enzymatic defect. Science 156, 1599-1602. doi: 10.1126/ science.156.3782.1599

Reiss, J., Gross-Hardt, S., Christensen, E., Schmidt, P., Mendel, R. R., and Schwarz, G. (2001). A mutation in the gene for the neurotransmitter receptor-clustering protein gephyrin causes a novel form of molybdenum cofactor deficiency. Am. J. Hum. Genet. 68, 208-213. doi: 10.1086/316941

Reiss, J., and Hahnewald, R. (2011). Molybdenum cofactor deficiency: mutations in GPHN, MOCS1, and MOCS2. Hum. Mutat. 32, 10-18. doi: 10.1002/humu. 21390

Rugarli, E. I., Langer, T., Akepati, V., Muller, E., Otto, A., Strauss, H., et al. (2012) Mitochondrial quality control: a matter of life and death for neurons. EMBO J. 31, 1336-1349. doi: 10.1038/emboj.2012.38

Schwahn, B. C., Van Spronsen, F. J., Belaidi, A. A., Bowhay, S., Christodoulou, J., Derks, T. G., et al. (2015). Efficacy and safety of cyclic pyranopterin monophosphate substitution in severe molybdenum cofactor deficiency type A: a prospective cohort study. Lancet 386, 1955-1963. doi: 10.1016/S01406736(15)00124-5

Schwarz, G. (2016). Molybdenum cofactor and human disease. Curr. Opin. Chem. Biol. 31, 179-187. doi: 10.1016/j.cbpa.2016.03.016

Schwarz, G., Mendel, R. R., and Ribbe, M. W. (2009). Molybdenum cofactors, enzymes and pathways. Nature 460, 839-847. doi: 10.1038/nature08302

Song, W., Chen, J., Petrilli, A., Liot, G., Klinglmayr, E., Zhou, Y., et al. (2011). Mutant huntingtin binds the mitochondrial fission GTPase dynamin-related protein-1 and increases its enzymatic activity. Nat. Med. 17, 377-382. doi: 10.1038/nm.2313

Song, W., Song, Y., Kincaid, B., Bossy, B., and Bossy-Wetzel, E. (2013). Mutant SOD1G93A triggers mitochondrial fragmentation in spinal cord motor neurons: neuroprotection by SIRT3 and PGC-1 $\alpha$. Neurobiol. Dis. 51, 72-81. doi: 10.1016/J.NBD.2012.07.004

Stipanuk, M. H., and Ueki, I. (2011). Dealing with methionine/homocysteine sulfur: cysteine metabolism to taurine and inorganic sulfur. J. Inherit. Metab. Dis. 34, 17-32. doi: 10.1007/s10545-009-9006-9

Suárez-Rivero, J., Villanueva-Paz, M., de la Cruz-Ojeda, P., de la Mata, M., Cotán, D., Oropesa-Ávila, M., et al. (2016). Mitochondrial dynamics in mitochondrial diseases. Diseases 5:1. doi: 10.3390/diseases5010001

Tiranti, V., D’Adamo, P., Briem, E., Ferrari, G., Mineri, R., Lamantea, E., et al. (2004). Ethylmalonic encephalopathy is caused by mutations in ETHE1, a gene encoding a mitochondrial matrix protein. Am. J. Hum. Genet. 74, 239-252. doi: $10.1086 / 381653$ 
Tiranti, V., Viscomi, C., Hildebrandt, T., Di Meo, I., Mineri, R., Tiveron, C., et al. (2009). Loss of ETHE1, a mitochondrial dioxygenase, causes fatal sulfide toxicity in ethylmalonic encephalopathy. Nat. Med. 15, 200-205. doi: 10.1038/ nm.1907

Verburg, J., and Hollenbeck, P. (2008). Mitochondrial membrane potential in axons increases with local nerve growth factor or semaphorin signaling. J. Neurosci. 28, 8306-8315. doi: 10.1523/JNEUROSCI.2614-08.2008

Vincent, A. S., Lim, B. G., Tan, J., Whiteman, M., Cheung, N. S., Halliwell, B., et al. (2004). Sulfite-mediated oxidative stress in kidney cells. Kidney Int. 65, 393-402. doi: 10.1111/J.1523-1755.2004.00391.X

Wai, T., and Langer, T. (2016). Mitochondrial dynamics and metabolic regulation. Trends Endocrinol. Metab. 27, 105-117. doi: 10.1016/J.TEM.2015.12.001

Wang, H., Song, P., Du, L., Tian, W., Yue, W., Liu, M., et al. (2011). Parkin ubiquitinates Drp1 for proteasome-dependent degradation: implication of dysregulated mitochondrial dynamics in Parkinson disease. J. Biol. Chem. 286, 11649-11658. doi: 10.1074/jbc.M110.144238

$\mathrm{Xu}$, J. (2005). Preparation, culture, and immortalization of mouse embryonic fibroblasts. Curr. Protoc. Mol. Biol. Chapter 28:Unit28.1. doi: 10.1002/ 0471142727.mb2801s70
Yi, M., Weaver, D., and Hajnóczky, G. (2004). Control of mitochondrial motility and distribution by the calcium signal. J. Cell Biol. 167, 661-672. doi: 10.1083/ jcb. 200406038

Zhang, X., Vincent, A. S., Halliwell, B., and Wong, K. P. (2004). A mechanism of sulfite neurotoxicity: direct inhibition of glutamate dehydrogenase. J. Biol. Chem. 279, 43035-43045. doi: 10.1074/jbc.M4027 59200

Conflict of Interest: The authors declare that the research was conducted in the absence of any commercial or financial relationships that could be construed as a potential conflict of interest.

Copyright (c) 2021 Mellis, Roeper, Misko, Kohl and Schwarz. This is an open-access article distributed under the terms of the Creative Commons Attribution License (CC BY). The use, distribution or reproduction in other forums is permitted, provided the original author(s) and the copyright owner(s) are credited and that the original publication in this journal is cited, in accordance with accepted academic practice. No use, distribution or reproduction is permitted which does not comply with these terms. 